\title{
Hospital Teaching Status and Trascatheter Aortic Valve Replacement Outcomes in the United States - Analysis of the National Inpatient Sample
}

${ }^{1}$ Tesfaye Telila MD, ${ }^{2}$ Emmanuel Akintoye MD, ${ }^{1}$ Tomo Ando MD, ${ }^{3}$ Obsinet Merid MD, ${ }^{1}$ Mohan Palla, ${ }^{1}$ Sagar Mallikethi-Reddy, ${ }^{4}$ Alexandros Briasoulis MD, ${ }^{1}$ Cindy Grines MD, ${ }^{1}$ Luis Afonso MD

${ }^{1}$ Division of Cardiology, Wayne State University/Detroit Medical center, Detroit, Michigan, United States.

${ }^{2}$ Detroit Medical Center, Division of Internal Medicine, Detroit, Michigan, United States

${ }^{3}$ University of Michigan Health Systems, Division of Internal Medicine.

${ }^{4}$ Mayo Clinic, Division of Cardiovascular Diseases, Rochester, Minnesota, United States.

Running title: Transcatheter aortic valve replacement outcomes and hospital teaching status

Keywords: Transcatheter aortic valve replacement, Hospital teaching status, Aortic stenosis

Word Count: 3079

Acknowledgments: None

Corresponding author:

Tesfaye Telila, MD

3990, John R, Detroit, Michigan, 48201, United States

Detroit Medical Center, Division of Cardiology

Phone: $313-745-2620$

Fax: $313-745-8643$

Email: tesfalex@gmail.com

This is the author manuscript accepted for publication and has undergone full peer review but has not been through the copyediting, typesetting, pagination and proofreading process, which may lead to differences between this version and the Version record. Please cite this article as doi:10.1002/ ccd.27236. 


\section{Abstract}

\section{Background:}

Evidence suggests that medical service offerings vary by hospital teaching status.

However, little is known about how these translate to patient outcomes. We therefore sought to evaluate this gap in knowledge in patients undergoing Transcatheter aortic valve replacement (TAVR) in the United States.

\section{Methods:}

This study was conducted using the National Inpatient Sample (NIS) in the United States from 2011 to 2014. Teaching status was classified, as teaching vs. non-teaching and endpoints were clinical outcomes, length of stay and cost. Procedure-related complications were identified via ICD-9 coding and analysis was performed via mixed effect model.

\section{Results:}

An estimated 33,790 TAVR procedures were performed in the U.S between 2011 and 2014, out of which $89.3 \%$ were in teaching hospitals. Mean (SD) age was 81.4 (8.5) and $47 \%$ were females. There was no significant difference between teaching versus nonteaching hospitals in regards to the primary outcome of in-hospital mortality and secondary outcomes of several cardiovascular and other end points except for a high rates of acute kidney injury (AKI) (OR: 1.34 [95\% CI, 1.04-1.72]) and lower rate for use of mechanical circulatory support devices in teaching vs. non-teaching centers. The mean length of stay was significantly higher in teaching hospitals (7.7 days) vs. non-teaching 
hospitals (6.8 days) $(\mathrm{P}=0.002)$ and so was the median cost of hospitalization (USD 50,814 vs. USD $48,787, \mathrm{P}=0.02$ ) for teaching vs. non-teaching centers.

\section{Conclusion:}

Most TAVR related short-term outcomes including all cause in-hospital mortality are about the same in teaching and non-teaching hospitals. However, AKI, length of hospital stay and TAVR related cost were significantly higher in teaching than non-teaching hospitals. There was more use of mechanical circulatory support in non-teaching than teaching hospitals.

\section{Introduction}

Transcatheter aortic valve replacement (TAVR) has become the standard of care in appropriate high and intermediate surgical risk patients with severe symptomatic aortic stenosis (1). Since its advent, the volume of patients undergoing TAVR has been increasing with concomitant improvement in outcomes and in-hospital mortality (2). However, certain complications such as vascular, cardiac, neurological, respiratory, renal, and even death remain (3). In a concerted attempt to mitigate complications and improve outcomes, a multidisciplinary heart team approach mainly comprising dedicated invasive and non-invasive cardiologists and cardiac surgeons in the establishment of a structural heart disease interventional program has been proposed (4). The large majority of such programs initially emerged in teaching centers and a few in non-teaching centers.

In-hospital mortality and rates of complications post TAVR were compared in teaching vs. non-teaching U.S centers in a prior study that reported a lower rate of inhospital complications in teaching vs. non-teaching centers (42\% vs. 50\% respectively, 
$\mathrm{P}<0.001)$ (5). However, these outcomes reflect only one-year data from 2012 when only very few non-teaching centers had TAVR programs. We sought to explore the evolution of this important theme in a more comprehensive four-year TAVR outcome analysis using data from the NIS registry (2011 to 2014).

\section{Methods}

\section{Data source}

Study was conducted using the NIS of the Health Care Utilization Project (HCUP). Details of the design and description of the NIS is available online (https://www.hcupus.ahrq.gov/nisoverview.jsp). In brief, NIS is the largest national database representative of all hospital discharges in the United States since 1998. It is a $20 \%$ stratified sampling of discharges from U.S. community hospitals, excluding rehabilitation and long-term acute care hospitals. Each year, over 7 million hospital stays are sampled nationwide which, when weighted, estimates more than 35 million hospitalizations per year.

\section{Patient population}

Our study utilizes information on adult patients (age $\geq 18$ years) who underwent TAVR across the United States between 2011 and 2014. Only patients who underwent endovascular (i.e. trans-femoral or trans-aortic) approach were included. Patients who underwent the transapical route were excluded due to available evidence indicating a significant difference in the pattern and rate of procedure-related outcomes compared to the transfemoral route $(6,7,8)$. In addition, recent evidence from the STS/ACC TAVR registry indicates a declining trend in the use of the transapical approach (9). Patients were identified using International Classification of Diseases-Ninth Revision, Clinical 
Modification (ICD-9-CM) procedure code 35.05. Patients were then classified into 2 major groups based on the teaching status as teaching and non-teaching centers. Baseline characteristics and post-procedure outcomes were compared between the two groups.

\section{Covariates}

Data on patient and hospital-level characteristics were provided for each patient in the NIS. However, identifiable variables were not included in order to preserve both patient and hospital privacy.. Patient-level factors including demographics, diagnoses, comorbidities, in-hospital procedures, disposition etc., as well as hospital level factors including bed-size, location, total number of hospitalizations etc. were available via the NIS database

\section{Endpoints}

The primary endpoint was in-hospital mortality defined as death at any time during the index hospitalization; secondary endpoints were post TAVR complications including acute myocardial infarction, neurologic complications, acute kidney injury, pacemaker placement, mechanical circulatory support, cardiac complications, vascular complications, hemorrhage requiring transfusion, sepsis, post-op venous thromboembolism, respiratory complications and non-routine discharge. In addition, our study also examined the length of hospital stay and hospital cost. As recommended by HCUP, the cost of hospitalization was indirectly estimated from the hospital charge that was reported. Information on cost was obtained from the hospital accounting reports collected by the Centers for Medicare and Medicaid Services (CMS) and these were used 
to generate hospital-specific cost-to-charge ratio that were applied to the hospital charge in order to estimate the cost.

\section{Statistical analysis}

Using the hospital-level trend (or discharge) weight provided for the NIS, we estimated national estimates including sum, rates and measure of central tendency. Comorbidity burden per hospitalization was quantified via the Elixhauser comorbidity index. To compare baseline patient- and hospital-level characteristics between non-teaching and teaching hospital, we used chi-square test for categorical variables, unpaired t-test for normally distributed continuous variables, and Wilcoxon rank-sum test for continuous variables with skewed distribution. Thereafter, we compared procedure-related outcomes between hospital teaching types using mixed effect models. This model enabled us to account for the potential correlation of observations within each hospital. As patient-level factors are nested within hospital-level factors, we built hierarchical model with unique hospital identification number as random effect in the model. Patient-level covariates including age, gender, Elixhauser comorbidity index, median household income, insurance status/expected payer, weekend admission; as well as hospital-level covariates including hospital bed size, hospital region and hospital location; and year of data collection were adjusted for in a multivariable analysis. Difference in cost and length of stay was evaluated via linear mixed model while other outcomes were evaluated via logistic mixed effect model. We performed complete case analysis using only observations with non-missing values for all the variables involved in the analyses, and analyses were performed using STATA 14 (StataCorp, College Station, Texas) with level of significance set at 0.05 . 


\section{Results}

From 2011-2014 a total of 33,790 transfemoral and transaortic TAVR procedures were performed in the United States. The overwhelming majority of TAVR procedures were performed in teaching centers $(89.3 \%)$ (Figure 1). There was a steady increase in the proportion of TAVR volumes in non-teaching hospitals from 2011 to 2013 but a slight decline from 2013 to 2014 . There was no significant difference in the mean age or gender distribution between teaching and non-teaching hospitals. More patients had Elixhauser comorbidity index $\geq 4(53.4 \%$ in teaching centers vs. $48.0 \%$ in nonteaching centers) (Table 1). There was no significant difference in the rates of primary outcome (all cause in-hospital mortality) (OR 1.03 and (95\% CI 0.70, 1.47) between teaching vs. non-teaching hospitals. The mean lengths of hospital stay (7.7 days vs. 6.7 days $(\mathrm{P}=0.002))$ and cost of hospitalizations (USD 50,814 vs. USD 48, $787(\mathrm{P}=0.02)$ were higher for teaching centers versus non-teaching centers (Figures 2). When we evaluated secondary endpoints, use of mechanical circulatory support was significantly lower in teaching hospital (OR 0.69 (95\% CI 0.47, 0.99) while rate of acute kidney injuries (AKI) was higher in teaching centers OR 1.34 (95\% CI 1.04, 1.72). However, there was no significant difference between the two hospital teaching statuses for all other secondary endpoints (Figures $3 \& 4$ ).

\section{Discussion}

The salient findings of our study may be summarized as follows: I) The primary outcome of all cause in-hospital mortality post TAVR was not significantly different between teaching and non-teaching hospitals, II) The secondary outcomes of post TAVR 
complications including acute myocardial infarction, neurologic complications, pacemaker placement, cardiac complications, vascular complications, hemorrhage requiring transfusion, sepsis, post-op venous thromboembolism, respiratory complications and non-routine discharge post TAVR did not differ significantly between teaching and non-teaching hospitals. III) Teaching hospital status was associated with higher risk of AKI and lower rates for use of mechanical circulatory support. IV) In teaching hospitals mean length of stay and median cost of hospitalization were significantly higher compared with non-teaching hospitals.

Our findings are consistent with the findings of a previous study by Pant et al, who analyzed 7,405 TAVR procedures from the NIS performed in 2012 and also found no significant difference in all-cause in-hospital mortality. However, unlike our study, the previous study from 2012 showed lower rates of TAVR related complications in teaching vs. non-teaching hospitals in the United States. Our study suggests that over the years, the difference in the rates of major TAVR related complications between teaching and nonteaching hospitals has narrowed considerably. This may be possibly explained by an increase in procedural volumes and operator expertise with ensuing reductions in complication rates, in non-teaching hospitals. This contrasting observation, deviating significantly from a prior report is a notable addition to the existing body of literature. Currently available data on the impact of teaching status on in-hospital outcomes after interventional cardiology procedures are limited. Our data demonstrated that the majority of TAVR patients were seen in teaching hospitals, consistent with a higher volume of procedures performed in these institutions. 
Operator and hospital volumes have been shown to be inversely related to complications post TAVR as indicated by a previous analyses of in-hospital outcomes (10). However, the Centers of Medicare \& Medicaid Services established specific procedural volume requirements that hospitals with and without TAVR experience must meet. Specifically, the initiation of a TAVR program requires $\geq 50$ aortic valve replacements, $\geq 1000$ catheterizations and $\geq 400$ percutaneous coronary interventions in the year prior to TAVR initiation (11). Therefore, teaching and non-teaching hospitals are now expected to have similar case volumes and experience with interventional cardiology and cardiothoracic surgery procedures in order to begin and continue a TAVR program.

Differences not only in volume but also implementation of guidelines and appropriate use criteria can positively impact patient-related outcomes. Registry data suggest variations in performance measures in teaching vs. non-teaching hospitals with greater adherence to standard guidelines for cardiovascular diseases, such as coronary artery disease and heart failure in teaching vs. non-teaching hospitals $(12,13)$. However, recent registry data suggest no significant association between hospital teaching status and adherence to guidelines among hospitals enrolled in the "Get With The Guidelines"Heart Failure program (14). These findings may be due to gradually increasing emphasis on implementation of protocolized guidelines, metrics and performance indicators for evaluation of comprehensive care delivery.

In addition to educational and research responsibilities, teaching hospitals are typically located in urban settings, serving populations with a lower socioeconomic and educational status and provide care as tertiary referral centers to patients with complicated cardiac conditions and multiple comorbidities. These factors in combination 
with the participation of less experienced physicians-in-training could plausibly lead to worse clinical outcomes and potentially slightly longer and more expensive hospitalizations as seen in our analysis. Although, we did not identify significant differences in the demographic characteristics or the comorbidity burden, our results should be interpreted with caution as many potential confounders may be unmeasured and missing.

The lack of difference in mortality between teaching and non-teaching hospitals was demonstrated in a meta-analysis of 132 observational cohort studies. However, the authors observed large between-study heterogeneity that precluded meaningful conclusion about other non-fatal outcomes (15). Therefore, based on the evidence summarized above, the focus should be shifted away from identification of differences in mortality and other hard cardiovascular outcomes between teaching and non-teaching hospitals but instead concentrated towards implementation of guidelines, appropriate use criteria, utilization of standardized quality measures and reduction of TAVR-related health care expenditures. Most importantly, although teaching in a healthcare facility does not seem to directly improve short-term patient outcomes, it is an integral part of cardiology, improves the level of knowledge and experience of future physicians in both teaching and non-teaching hospitals and as such should be strongly encouraged and supported.

Potential limitations of our study should also be considered. First, due to the observational nature of the study, we cannot claim a causal link between teaching status and any of the endpoints because other unmeasured covariates may have contributed to this association. However, we limited the potential bias in the associations by adjusting 
for multiple patient- and hospital-level covariates that might have confounded the associations. Second, our analysis was limited to in-patient data and, hence, we are not able to make any inference about patient outcomes in the immediate post-discharge period that may be related to in-patient care. Despite these limitations, NIS remains a large, easily accessible dataset that has been widely used to estimate national trends in inpatient outcomes.

P.

\section{Conclusion}

We found no substantial differences between teaching and non-teaching hospitals in regards to in-hospital mortality and other procedure-related complications post TAVR. However, we identified high rates of post TAVR AKI, longer hospital stay and higher cost of hospitalization in teaching centers. The use of mechanical circulatory support post TAVR was more frequent in non-teaching compared to teaching hospitals.

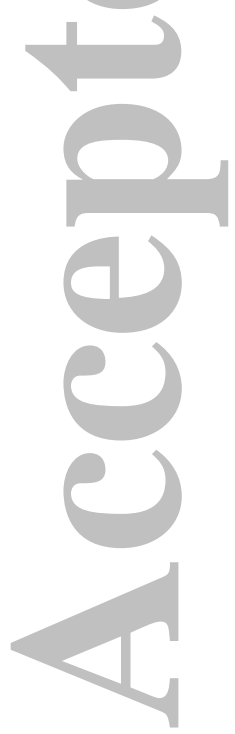




\section{References}

1. Otto CM, Kumbhani DJ, Alexander KP, Calhoon JH, Desai MY, Kaul S, Lee JC, Ruiz CE, Vassileva CM. 2017 ACC Expert Consensus Decision Pathway for Transcatheter Aortic Valve Replacement in the Management of Adults With Aortic Stenosis: A Report of the American College of Cardiology Task Force on Clinical Expert Consensus Documents. J Am Coll Cardiol. 2017;69(10):13131346.

2. Brennan JM, Holmes DR, Sherwood MW, Edwards FH, Carroll JD, Grover FL, Tuzcu EM, Thourani V, Brindis RG, Shahian DM, Svensson LG, O'Brien SM, Shewan CM, Hewitt K, Gammie JS, Rumsfeld JS, Peterson ED, Mack MJ. The association of transcatheter aortic valve replacement availability and hospital aortic valve replacement volume and mortality in the United States. Ann Thorac Surg. 2014; 98(6): 2016-22.

3. Fassa AA, Himbert D, Vahanian A. Mechanisms and management of TAVRrelated complications. Nat Rev Cardiol. 2013; 10:685-695.

4. Tommaso CL, Bolman RM, III, Feldman T. Multisociety (AATS, ACCF, SCAI, and STS) expert consensus statement: Operator and institutional requirements for transcatheter valve repair and replacement, part 1: Transcatheter aortic valve replacement. J Thorac Cardiovasc Surg. 2012; 143:1254-1263.

5. Pant S, Patel S, Golwala H, Patel N, Pandey A, Badheka A, Agnihotri K, Patel N, Deshmukh A, Flaherty MP. Transcatheter Aortic Valve Replacement Complication Rates in Teaching Vs Non-Teaching Centers in the United States. $J$ Invasive Cardiol. 2016;28(2):67-70. 
6. Biancari, F.Rosato, S.D'Errigo, P.Ranucci, M.Onorati, F.Barbanti, M. Santini, F. Tamburino, C. Santoro, G. Grossi, C. Covello, R. D. Ventura, M. Fusco, D. Seccareccia, F. OBSERVANT Research Group Immediate and Intermediate Outcome After Transapical Versus Transfemoral Transcatheter Aortic Valve Replacement. Am J Cardiol. 2016;117(2):245-251.

7. Walther T, Kempfert J. Transapical vs. transfemoral aortic valve implantation: Which approach for which patient, from a surgeon's standpoint. Ann Cardiothorac Surg. 2012;1(2):216-219.

8. Zhao A, Minhui H, Li X, Zhiyun X. A meta-analysis of transfemoral versus transapical transcatheter aortic valve implantation on 30-day and 1-year outcomes. Heart Surg Forum. 2015;18(4):E161-166.

9. Grover, F. L. Vemulapalli, S. Carroll, J. D. Edwards, F. H. Mack, M. J. Thourani, V. H. Brindis, R. G. Shahian, D. M. Ruiz, C. E. Jacobs, J. P. Hanzel, G. Bavaria, J. E. Tuzcu, E. M. Peterson, E. D. Fitzgerald, S. Kourtis, M. Michaels, J. Christensen, B. Seward, W. F. Hewitt, K. Holmes, D. R. STS/ACC TVT Registry 2016 Annual Report of The Society of Thoracic Surgeons/American College of Cardiology Transcatheter Valve Therapy Registry. J Am Coll Cardiol. 2017;69(10):1215-1230.

10. Badheka AO, Patel NJ, Panaich SS, et al. Effect of hospital volume on outcomes of transcatheter aortic valve implantation. Am J Cardiol 2015; $116: 587-594$ 
11. Center for Medicare and Medicaid Services 2016. https://www.cms.gov/Outreach-and-Education/Medicare-Learning-NetworkMLN/MLNMattersArticles/downloads/SE1515.

12. Tam LM, Fonarow GC, Bhatt DL, Grau-Sepulveda MV, Hernandez AF, Peterson ED, Schwamm LH, Giugliano RP; GWTG Steering Committee and Investigators. Achievement of guideline-concordant care and in-hospital outcomes in patients with coronary artery disease in teaching and nonteaching hospitals: results from the Get With The Guidelines-Coronary Artery Disease program. Circ Cardiovasc Qual Outcomes. 2013; 6:58-65.

13. Fonarow GC, Yancy CW, Heywood JT; ADHERE Scientific Advisory Committee, Study Group, and Investigators. Adherence to heart failure quality-of-care indicators in US hospitals: analysis of the ADHERE Registry. Arch Intern Med. 2005; 165:1469-1477.

14. Patel DB, Shah RM, Bhatt DL, Liang L, Schulte PJ, DeVore AD, Hernandez AF, Heidenreich PA, Yancy CW, Fonarow GC. Guideline-Appropriate Care and In-Hospital Outcomes in Patients With Heart Failure in Teaching and Nonteaching Hospitals: Findings From Get With The Guidelines-Heart Failure. Circ Cardiovasc Qual Outcomes. 2016; 9(6): 757-766.

15. Papanikolaou PN, Christidi GD, Ioannidis JP. Patient outcomes with teaching versus nonteaching healthcare: a systematic review. PLoS Med. 2006;3(9):e341 


\section{$\underline{\text { Figure Legends }}$}

Table 1. Baseline characteristics of patient hospitalized for TAVR, stratified by hospital teaching status.

TAVR: Transcatheter aortic valve replacement

PVD: Peripheral vascular disease

Figure 1. Trend in percentage of TAVR procedures performed per year between nonteaching and teaching hospitals (2011-2014).

TAVR: Transcatheter aortic valve replacement

Figure 2. Cost of hospitalization for TAVR between hospital teaching status ( $p$ for difference $=0.02$ )

USD: United States dollars

Figure 3: Rates (\%) of TAVR-associated complications

Acute MI: Acute Myocardial infarction

DVT/PE: Deep vein thrombosis/Pulmonary embolism

Figure 4. Comparing TAVR-associated complications between teaching and nonteaching hospital

DVT/PE: Deep vein thrombosis/Pulmonary embolism 
Table 1. Baseline characteristics of patients hospitalized for transcatheter aortic valve replacement, stratified by hospital teaching status

\begin{tabular}{|c|c|c|c|c|}
\hline 5 & Total & Non-teaching & Teaching & P value \\
\hline Unweighted No. (\%) & $6,778(100)$ & $727(10.7)$ & $6,051(89.3)$ & \\
\hline Weighted No. (\%) & $33,790(100)$ & $3,621(10.7)$ & $30,169(89.3)$ & \\
\hline Age, mean (SD) & $81.4(8.5)$ & $81.5(8.3)$ & $81.4(8.6)$ & 0.59 \\
\hline Female, \% & 46.1 & 44.8 & 46.1 & 0.49 \\
\hline \multicolumn{5}{|l|}{ Race, \% } \\
\hline White & 87.4 & 92.0 & 86.8 & \\
\hline Black & 4.1 & 2.5 & 4.4 & \\
\hline Hispanic & 3.4 & 1.3 & 3.6 & $<0.001$ \\
\hline Asia & 1.1 & 1.3 & 1.0 & \\
\hline Others & 4.1 & 2.9 & 4.2 & \\
\hline Obesity, \% & 14.6 & 13.5 & 14.7 & 0.37 \\
\hline Diabetes, \% & 34.9 & 33.8 & 35.0 & 0.55 \\
\hline PVD, \% & 28.2 & 23.5 & 28.7 & 0.003 \\
\hline Hypertension, \% & 79.6 & 76.6 & 79.9 & 0.04 \\
\hline Congestive heart failure, $\%$ & 12.8 & 11.4 & 13.0 & 0.24 \\
\hline Chronic lung disease, \% & 32.6 & 29.0 & 33.0 & 0.03 \\
\hline Renal failure, $\%$ & 36.7 & 39.8 & 36.3 & 0.06 \\
\hline Liver disease, $\%$ & 2.7 & 2.1 & 2.8 & 0.26 \\
\hline Cerebrovascular disease, $\%$ & 10.0 & 9.9 & 10.1 & 0.86 \\
\hline Rheumatologic disease, $\%$ & 4.8 & 4.8 & 4.8 & 0.98 \\
\hline
\end{tabular}




\begin{tabular}{|c|c|c|c|c|}
\hline \multicolumn{5}{|c|}{$\begin{array}{l}\text { Elixhauser comorbidity index, } \\
\%\end{array}$} \\
\hline $0-1$ & 9.6 & 12.4 & 9.2 & \\
\hline $2-3$ & 37.6 & 39.6 & 37.4 & 0.004 \\
\hline$\geq 4$ & 52.8 & 48.0 & 53.4 & \\
\hline \multicolumn{5}{|c|}{ Median household income, \% } \\
\hline $1^{\text {st }}$ quartile & 20.7 & 22.0 & 20.6 & \\
\hline $2^{\text {nd }}$ quartile & 24.9 & 31.5 & 24.1 & $<0.001$ \\
\hline $3^{\text {rd }}$ quartile & 25.5 & 22.7 & 25.9 & \\
\hline $4^{\text {th }}$ quartile & 28.9 & 23.8 & 29.5 & \\
\hline
\end{tabular}

Hospital bed size, \%

\begin{tabular}{|c|c|c|c|c|}
\hline Small & 4.3 & 12.8 & 3.3 & \\
\hline Medium & 15.5 & 23.5 & 14.5 & $<0.001$ \\
\hline Large & 80.2 & 63.7 & 82.2 & \\
\hline \multicolumn{5}{|l|}{ Hospital region, \% } \\
\hline Northeast & 25.7 & 13.9 & 27.1 & \\
\hline Midwest & 22.3 & 25.6 & 22.0 & $<0.001$ \\
\hline South & 34.4 & 41.1 & 33.6 & \\
\hline West & 17.6 & 19.4 & 17.3 & \\
\hline
\end{tabular}


Figure 1. Trend in percentage of TAVR procedures performed per year between non-teaching and teaching hospitals (2011-2014)

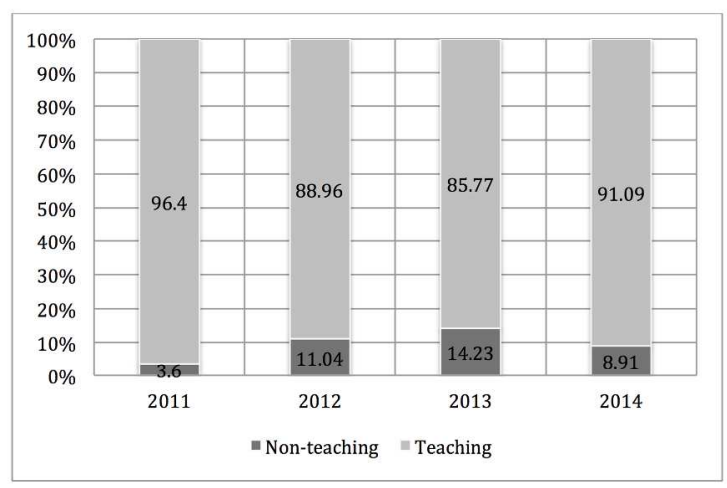

Figure 1. Trend in percentage of TAVR procedures performed per year between non-teaching and teaching hospitals (2011-2014).

TAVR: Transcatheter aortic valve replacement

$215 \times 279 \mathrm{~mm}(300 \times 300 \mathrm{DPI})$

Catheterization and Cardiovascular Interventions

This article is protected by copyright. All rights reserved. 
Figure 2. Cost of hospitalization for TAVR between hospital teaching status ( $p$ for difference $=0.02$ )

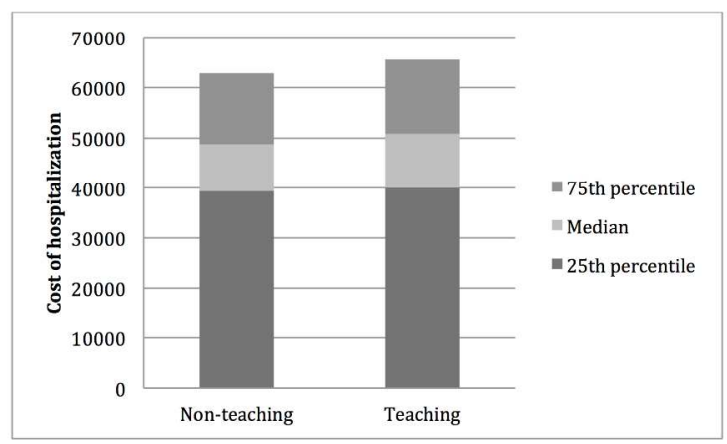

Values (USD) for Figure 2.

\begin{tabular}{|l|l|l|}
\hline & $\begin{array}{l}\text { Non- } \\
\text { teaching }\end{array}$ & Teaching \\
\hline $\begin{array}{l}\text { 25th } \\
\text { percentile }\end{array}$ & 39341 & 40024 \\
\hline Median & 48787 & 50814 \\
\hline $\begin{array}{l}75 \text { th } \\
\text { percentile }\end{array}$ & 62796 & 65552 \\
\hline
\end{tabular}

Figure 2. Cost of hospitalization for TAVR between hospital teaching status ( $p$ for difference $=0.02$ ) USD: United States dollars

$215 \times 279 \mathrm{~mm}(300 \times 300 \mathrm{DPI})$ 
Figure 3: Rates (\%) of TAVR-associated complications

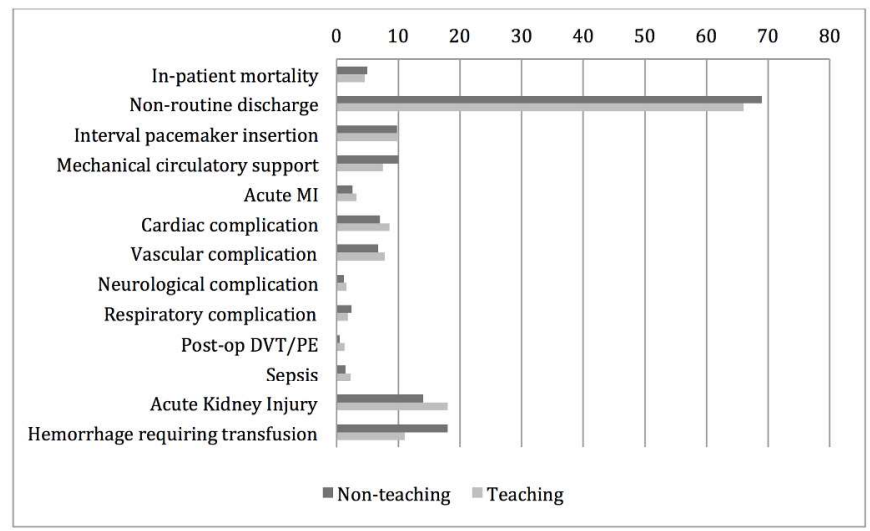

Values for figure 3

\begin{tabular}{|l|l|l|}
\hline & Non-teaching & Teaching \\
\hline In-patient mortality & 4.94 & 4.59 \\
\hline Non-routine discharge & 69 & 66 \\
\hline Interval pacemaker insertion & 9.8 & 10 \\
\hline Mechanical circulatory support & 10 & 7.5 \\
\hline Acute MI & 2.6 & 3.2 \\
\hline Cardiac complication & 7 & 8.6 \\
\hline Vascular complication & 6.7 & 7.8 \\
\hline Neurological complication & 1.2 & 1.6 \\
\hline Respiratory complication & 2.4 & 1.8 \\
\hline Post-op DVT/PE & 0.47 & 1.3 \\
\hline Sepsis & 1.4 & 2.3 \\
\hline Acute Kidney Injury & 14 & 18 \\
\hline Hemorrhage requiring transfusion & 18 & 11 \\
\hline
\end{tabular}

Figure 3: Rates (\%) of TAVR-associated complications Acute MI: Acute Myocardial infarction

$215 \times 279 \mathrm{~mm}(300 \times 300 \mathrm{DPI})$ 
Figure 4. Comparing TAVR-associated complications between teaching and non-teaching hospital

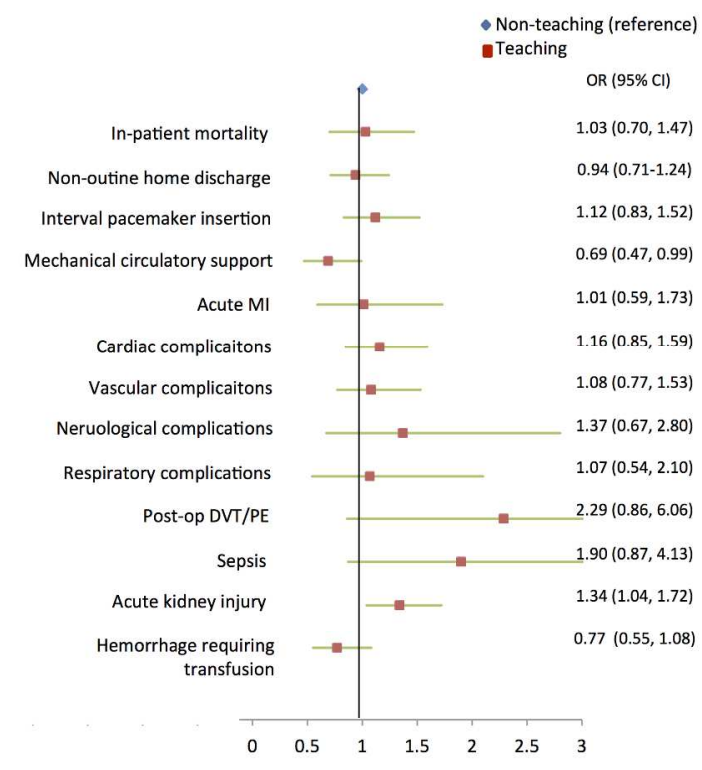

Figure 4. Comparing TAVR-associated complications between teaching and non-teaching hospital DVT/PE: Deep vein thrombosis/Pulmonary embolism $215 \times 279 \mathrm{~mm}(300 \times 300 \mathrm{DPI})$ 


\title{
Hospital Teaching Status and Trascatheter Aortic Valve Replacement Outcomes in the United States - Analysis of the National Inpatient Sample
}

\author{
${ }^{1}$ Tesfaye Telila MD, ${ }^{2}$ Emmanuel Akintoye MD, ${ }^{1}$ Tomo Ando MD, ${ }^{3}$ Obsinet Merid
} MD, ${ }^{1}$ Mohan Palla, ${ }^{1}$ Sagar Mallikethi-Reddy, ${ }^{4}$ Alexandros Briasoulis MD, ${ }^{1}$ Cindy Grines MD, ${ }^{1}$ Luis Afonso MD

${ }^{1}$ Division of Cardiology, Wayne State University/Detroit Medical center, Detroit, Michigan, United States.

${ }^{2}$ Detroit Medical Center, Division of Internal Medicine, Detroit, Michigan, United States

${ }^{3}$ University of Michigan Health Systems, Division of Internal Medicine.

${ }^{4}$ Mayo Clinic, Division of Cardiovascular Diseases, Rochester, Minnesota, United States.

Running title: Transcatheter aortic valve replacement outcomes and hospital teaching status

Keywords: Transcatheter aortic valve replacement, Hospital teaching status, Aortic stenosis

Word Count: 3079

Acknowledgments: None

Corresponding author:

Tesfaye Telila, MD

3990, John R, Detroit, Michigan, 48201, United States

Detroit Medical Center, Division of Cardiology

Phone: $313-745-2620$

Fax: $313-745-8643$

Email: tesfalex@gmail.com 


\section{Abstract}

\section{Background:}

Evidence suggests that medical service offerings vary by hospital teaching status.

However, little is known about how these translate to patient outcomes. We therefore sought to evaluate this gap in knowledge in patients undergoing Transcatheter aortic valve replacement (TAVR) in the United States.

\section{Methods:}

This study was conducted using the National Inpatient Sample (NIS) in the United States from 2011 to 2014. Teaching status was classified, as teaching vs. non-teaching and endpoints were clinical outcomes, length of stay and cost. Procedure-related complications were identified via ICD-9 coding and analysis was performed via mixed effect model.

\section{Results:}

An estimated 33,790 TAVR procedures were performed in the U.S between 2011 and 2014, out of which $89.3 \%$ were in teaching hospitals. Mean (SD) age was 81.4 (8.5) and 47\% were females. There was no significant difference between teaching versus nonteaching hospitals in regards to the primary outcome of in-hospital mortality and secondary outcomes of several cardiovascular and other end points except for a high rates of acute kidney injury (AKI) (OR: 1.34 [95\% CI, 1.04-1.72]) and lower rate for use of mechanical circulatory support devices in teaching vs. non-teaching centers. The mean length of stay was significantly higher in teaching hospitals (7.7 days) vs. non-teaching 
hospitals (6.8 days) $(\mathrm{P}=0.002)$ and so was the median cost of hospitalization (USD 50,814 vs. USD $48,787, \mathrm{P}=0.02$ ) for teaching vs. non-teaching centers.

\section{Conclusion:}

Most TAVR related short-term outcomes including all cause in-hospital mortality are about the same in teaching and non-teaching hospitals. However, AKI, length of hospital stay and TAVR related cost were significantly higher in teaching than non-teaching hospitals. There was more use of mechanical circulatory support in non-teaching than teaching hospitals.

\section{Introduction}

Transcatheter aortic valve replacement (TAVR) has become the standard of care in appropriate high and intermediate surgical risk patients with severe symptomatic aortic stenosis (1). Since its advent, the volume of patients undergoing TAVR has been increasing with concomitant improvement in outcomes and in-hospital mortality (2). However, certain complications such as vascular, cardiac, neurological, respiratory, renal, and even death remain (3). In a concerted attempt to mitigate complications and improve outcomes, a multidisciplinary heart team approach mainly comprising dedicated invasive and non-invasive cardiologists and cardiac surgeons in the establishment of a structural heart disease interventional program has been proposed (4). The large majority of such programs initially emerged in teaching centers and a few in non-teaching centers.

In-hospital mortality and rates of complications post TAVR were compared in teaching vs. non-teaching U.S centers in a prior study that reported a lower rate of inhospital complications in teaching vs. non-teaching centers (42\% vs. 50\% respectively, 
$\mathrm{P}<0.001)$ (5). However, these outcomes reflect only one-year data from 2012 when only very few non-teaching centers had TAVR programs. We sought to explore the evolution of this important theme in a more comprehensive four-year TAVR outcome analysis using data from the NIS registry (2011 to 2014).

\section{Methods}

\section{Data source}

Study was conducted using the NIS of the Health Care Utilization Project (HCUP). Details of the design and description of the NIS is available online (https://www.hcupus.ahrq.gov/nisoverview.jsp). In brief, NIS is the largest national database representative of all hospital discharges in the United States since 1998. It is a $20 \%$ stratified sampling of discharges from U.S. community hospitals, excluding rehabilitation and long-term acute care hospitals. Each year, over 7 million hospital stays are sampled nationwide which, when weighted, estimates more than 35 million hospitalizations per year.

\section{Patient population}

Our study utilizes information on adult patients (age $\geq 18$ years) who underwent TAVR across the United States between 2011 and 2014. Only patients who underwent endovascular (i.e. trans-femoral or trans-aortic) approach were included. Patients who underwent the transapical route were excluded due to available evidence indicating a significant difference in the pattern and rate of procedure-related outcomes compared to the transfemoral route $(6,7,8)$. In addition, recent evidence from the STS/ACC TAVR registry indicates a declining trend in the use of the transapical approach (9). Patients were identified using International Classification of Diseases-Ninth Revision, Clinical 
Modification (ICD-9-CM) procedure code 35.05. Patients were then classified into 2 major groups based on the teaching status as teaching and non-teaching centers. Baseline characteristics and post-procedure outcomes were compared between the two groups.

\section{Covariates}

Data on patient and hospital-level characteristics were provided for each patient in the NIS. However, identifiable variables were not included in order to preserve both patient and hospital privacy.. Patient-level factors including demographics, diagnoses, comorbidities, in-hospital procedures, disposition etc., as well as hospital level factors including bed-size, location, total number of hospitalizations etc. were available via the NIS database

\section{Endpoints}

The primary endpoint was in-hospital mortality defined as death at any time during the index hospitalization; secondary endpoints were post TAVR complications including acute myocardial infarction, neurologic complications, acute kidney injury, pacemaker placement, mechanical circulatory support, cardiac complications, vascular complications, hemorrhage requiring transfusion, sepsis, post-op venous thromboembolism, respiratory complications and non-routine discharge. In addition, our study also examined the length of hospital stay and hospital cost. As recommended by HCUP, the cost of hospitalization was indirectly estimated from the hospital charge that was reported. Information on cost was obtained from the hospital accounting reports collected by the Centers for Medicare and Medicaid Services (CMS) and these were used 
to generate hospital-specific cost-to-charge ratio that were applied to the hospital charge in order to estimate the cost.

\section{Statistical analysis}

Using the hospital-level trend (or discharge) weight provided for the NIS, we estimated national estimates including sum, rates and measure of central tendency. Comorbidity burden per hospitalization was quantified via the Elixhauser comorbidity index. To compare baseline patient- and hospital-level characteristics between non-teaching and teaching hospital, we used chi-square test for categorical variables, unpaired t-test for normally distributed continuous variables, and Wilcoxon rank-sum test for continuous variables with skewed distribution. Thereafter, we compared procedure-related outcomes between hospital teaching types using mixed effect models. This model enabled us to account for the potential correlation of observations within each hospital. As patient-level factors are nested within hospital-level factors, we built hierarchical model with unique hospital identification number as random effect in the model. Patient-level covariates including age, gender, Elixhauser comorbidity index, median household income, insurance status/expected payer, weekend admission; as well as hospital-level covariates including hospital bed size, hospital region and hospital location; and year of data collection were adjusted for in a multivariable analysis. Difference in cost and length of stay was evaluated via linear mixed model while other outcomes were evaluated via logistic mixed effect model. We performed complete case analysis using only observations with non-missing values for all the variables involved in the analyses, and analyses were performed using STATA 14 (StataCorp, College Station, Texas) with level of significance set at 0.05 . 


\section{Results}

From 2011-2014 a total of 33,790 transfemoral and transaortic TAVR procedures were performed in the United States. The overwhelming majority of TAVR procedures were performed in teaching centers $(89.3 \%)$ (Figure 1). There was a steady increase in the proportion of TAVR volumes in non-teaching hospitals from 2011 to 2013 but a slight decline from 2013 to 2014 . There was no significant difference in the mean age or gender distribution between teaching and non-teaching hospitals. More patients had Elixhauser comorbidity index $\geq 4(53.4 \%$ in teaching centers vs. $48.0 \%$ in nonteaching centers) (Table 1). There was no significant difference in the rates of primary outcome (all cause in-hospital mortality) (OR 1.03 and $(95 \%$ CI $0.70,1.47)$ between teaching vs. non-teaching hospitals. The mean lengths of hospital stay ( 7.7 days vs. 6.7 days $(\mathrm{P}=0.002))$ and cost of hospitalizations (USD 50,814 vs. USD 48, $787(\mathrm{P}=0.02)$ were higher for teaching centers versus non-teaching centers (Figures 2). When we evaluated secondary endpoints, use of mechanical circulatory support was significantly lower in teaching hospital (OR 0.69 (95\% CI 0.47, 0.99) while rate of acute kidney injuries (AKI) was higher in teaching centers OR 1.34 (95\% CI 1.04, 1.72). However, there was no significant difference between the two hospital teaching statuses for all other secondary endpoints (Figures $3 \& 4$ ).

\section{Discussion}

The salient findings of our study may be summarized as follows: I) The primary outcome of all cause in-hospital mortality post TAVR was not significantly different between teaching and non-teaching hospitals, II) The secondary outcomes of post TAVR 
complications including acute myocardial infarction, neurologic complications, pacemaker placement, cardiac complications, vascular complications, hemorrhage requiring transfusion, sepsis, post-op venous thromboembolism, respiratory complications and non-routine discharge post TAVR did not differ significantly between teaching and non-teaching hospitals. III) Teaching hospital status was associated with higher risk of AKI and lower rates for use of mechanical circulatory support. IV) In teaching hospitals mean length of stay and median cost of hospitalization were significantly higher compared with non-teaching hospitals.

Our findings are consistent with the findings of a previous study by Pant et al, who analyzed 7,405 TAVR procedures from the NIS performed in 2012 and also found no significant difference in all-cause in-hospital mortality. However, unlike our study, the previous study from 2012 showed lower rates of TAVR related complications in teaching vs. non-teaching hospitals in the United States. Our study suggests that over the years, the difference in the rates of major TAVR related complications between teaching and nonteaching hospitals has narrowed considerably. This may be possibly explained by an increase in procedural volumes and operator expertise with ensuing reductions in complication rates, in non-teaching hospitals. This contrasting observation, deviating significantly from a prior report is a notable addition to the existing body of literature. Currently available data on the impact of teaching status on in-hospital outcomes after interventional cardiology procedures are limited. Our data demonstrated that the majority of TAVR patients were seen in teaching hospitals, consistent with a higher volume of procedures performed in these institutions. 
Operator and hospital volumes have been shown to be inversely related to complications post TAVR as indicated by a previous analyses of in-hospital outcomes (10). However, the Centers of Medicare \& Medicaid Services established specific procedural volume requirements that hospitals with and without TAVR experience must meet. Specifically, the initiation of a TAVR program requires $\geq 50$ aortic valve replacements, $\geq 1000$ catheterizations and $\geq 400$ percutaneous coronary interventions in the year prior to TAVR initiation (11). Therefore, teaching and non-teaching hospitals are now expected to have similar case volumes and experience with interventional cardiology and cardiothoracic surgery procedures in order to begin and continue a TAVR program.

Differences not only in volume but also implementation of guidelines and appropriate use criteria can positively impact patient-related outcomes. Registry data suggest variations in performance measures in teaching vs. non-teaching hospitals with greater adherence to standard guidelines for cardiovascular diseases, such as coronary artery disease and heart failure in teaching vs. non-teaching hospitals $(12,13)$. However, recent registry data suggest no significant association between hospital teaching status and adherence to guidelines among hospitals enrolled in the "Get With The Guidelines"Heart Failure program (14). These findings may be due to gradually increasing emphasis on implementation of protocolized guidelines, metrics and performance indicators for evaluation of comprehensive care delivery.

In addition to educational and research responsibilities, teaching hospitals are typically located in urban settings, serving populations with a lower socioeconomic and educational status and provide care as tertiary referral centers to patients with complicated cardiac conditions and multiple comorbidities. Although, we did not identify 
significant differences in the demographic characteristics or the comorbidity burden, our results should be interpreted with caution as many potential confounders may be unmeasured and missing.

The lack of difference in mortality between teaching and non-teaching hospitals was demonstrated in a meta-analysis of 132 observational cohort studies. However, the authors observed large between-study heterogeneity that precluded meaningful conclusion about other non-fatal outcomes (15). Therefore, based on the evidence summarized above, the focus should be shifted away from identification of differences in mortality and other hard cardiovascular outcomes between teaching and non-teaching hospitals but instead concentrated towards implementation of guidelines, appropriate use criteria, utilization of standardized quality measures and reduction of TAVR-related health care expenditures. Most importantly, although teaching in a healthcare facility does not seem to directly improve short-term patient outcomes, it is an integral part of cardiology, improves the level of knowledge and experience of future physicians in both teaching and non-teaching hospitals and as such should be strongly encouraged and supported.

Potential limitations of our study should also be considered. First, due to the observational nature of the study, we cannot claim a causal link between teaching status and any of the endpoints because other unmeasured covariates may have contributed to this association. However, we limited the potential bias in the associations by adjusting for multiple patient- and hospital-level covariates that might have confounded the associations. Second, our analysis was limited to in-patient data and, hence, we are not able to make any inference about patient outcomes in the immediate post-discharge 
period that may be related to in-patient care. Despite these limitations, NIS remains a large, easily accessible dataset that has been widely used to estimate national trends in inpatient outcomes.

\section{Conclusion}

We found no substantial differences between teaching and non-teaching hospitals in regards to in-hospital mortality and other procedure-related complications post TAVR. However, we identified high rates of post TAVR AKI, longer hospital stay and higher cost of hospitalization in teaching centers. The use of mechanical circulatory support post TAVR was more frequent in non-teaching compared to teaching hospitals.

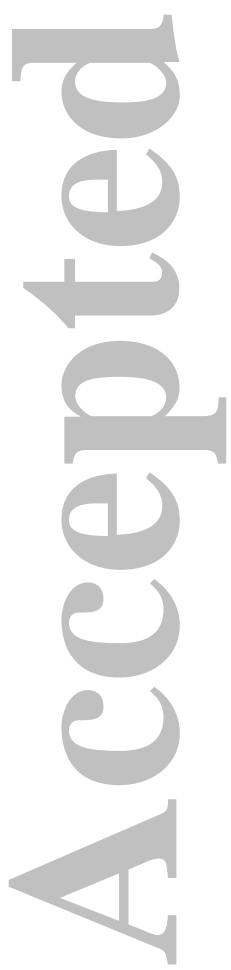




\section{References}

1. Otto CM, Kumbhani DJ, Alexander KP, Calhoon JH, Desai MY, Kaul S, Lee JC, Ruiz CE, Vassileva CM. 2017 ACC Expert Consensus Decision Pathway for Transcatheter Aortic Valve Replacement in the Management of Adults With Aortic Stenosis: A Report of the American College of Cardiology Task Force on Clinical Expert Consensus Documents. J Am Coll Cardiol. 2017;69(10):13131346.

2. Brennan JM, Holmes DR, Sherwood MW, Edwards FH, Carroll JD, Grover FL, Tuzcu EM, Thourani V, Brindis RG, Shahian DM, Svensson LG, O'Brien SM, Shewan CM, Hewitt K, Gammie JS, Rumsfeld JS, Peterson ED, Mack MJ. The association of transcatheter aortic valve replacement availability and hospital aortic valve replacement volume and mortality in the United States. Ann Thorac Surg. 2014; 98(6): 2016-22.

3. Fassa AA, Himbert D, Vahanian A. Mechanisms and management of TAVRrelated complications. Nat Rev Cardiol. 2013; 10:685-695.

4. Tommaso CL, Bolman RM, III, Feldman T. Multisociety (AATS, ACCF, SCAI, and STS) expert consensus statement: Operator and institutional requirements for transcatheter valve repair and replacement, part 1: Transcatheter aortic valve replacement. J Thorac Cardiovasc Surg. 2012; 143:1254-1263.

5. Pant S, Patel S, Golwala H, Patel N, Pandey A, Badheka A, Agnihotri K, Patel N, Deshmukh A, Flaherty MP. Transcatheter Aortic Valve Replacement Complication Rates in Teaching Vs Non-Teaching Centers in the United States. $J$ Invasive Cardiol. 2016;28(2):67-70. 
6. Biancari, F.Rosato, S.D'Errigo, P.Ranucci, M.Onorati, F.Barbanti, M. Santini, F. Tamburino, C. Santoro, G. Grossi, C. Covello, R. D. Ventura, M. Fusco, D. Seccareccia, F. OBSERVANT Research Group Immediate and Intermediate Outcome After Transapical Versus Transfemoral Transcatheter Aortic Valve Replacement. Am J Cardiol. 2016;117(2):245-251.

7. Walther T, Kempfert J. Transapical vs. transfemoral aortic valve implantation: Which approach for which patient, from a surgeon's standpoint. Ann Cardiothorac Surg. 2012;1(2):216-219.

8. Zhao A, Minhui H, Li X, Zhiyun X. A meta-analysis of transfemoral versus transapical transcatheter aortic valve implantation on 30-day and 1-year outcomes. Heart Surg Forum. 2015;18(4):E161-166.

9. Grover, F. L. Vemulapalli, S. Carroll, J. D. Edwards, F. H. Mack, M. J. Thourani, V. H. Brindis, R. G. Shahian, D. M. Ruiz, C. E. Jacobs, J. P. Hanzel, G. Bavaria, J. E. Tuzcu, E. M. Peterson, E. D. Fitzgerald, S. Kourtis, M. Michaels, J. Christensen, B. Seward, W. F. Hewitt, K. Holmes, D. R. STS/ACC TVT Registry 2016 Annual Report of The Society of Thoracic Surgeons/American College of Cardiology Transcatheter Valve Therapy Registry. J Am Coll Cardiol. 2017;69(10):1215-1230.

10. Badheka AO, Patel NJ, Panaich SS, et al. Effect of hospital volume on outcomes of transcatheter aortic valve implantation. Am J Cardiol 2015; $116: 587-594$ 
11. Center for Medicare and Medicaid Services 2016. https://www.cms.gov/Outreach-and-Education/Medicare-Learning-NetworkMLN/MLNMattersArticles/downloads/SE1515.

12. Tam LM, Fonarow GC, Bhatt DL, Grau-Sepulveda MV, Hernandez AF, Peterson ED, Schwamm LH, Giugliano RP; GWTG Steering Committee and Investigators. Achievement of guideline-concordant care and in-hospital outcomes in patients with coronary artery disease in teaching and nonteaching hospitals: results from the Get With The Guidelines-Coronary Artery Disease program. Circ Cardiovasc Qual Outcomes. 2013; 6:58-65.

13. Fonarow GC, Yancy CW, Heywood JT; ADHERE Scientific Advisory Committee, Study Group, and Investigators. Adherence to heart failure quality-of-care indicators in US hospitals: analysis of the ADHERE Registry. Arch Intern Med. 2005; 165:1469-1477.

14. Patel DB, Shah RM, Bhatt DL, Liang L, Schulte PJ, DeVore AD, Hernandez AF, Heidenreich PA, Yancy CW, Fonarow GC. Guideline-Appropriate Care and In-Hospital Outcomes in Patients With Heart Failure in Teaching and Nonteaching Hospitals: Findings From Get With The Guidelines-Heart Failure. Circ Cardiovasc Qual Outcomes. 2016; 9(6): 757-766.

15. Papanikolaou PN, Christidi GD, Ioannidis JP. Patient outcomes with teaching versus nonteaching healthcare: a systematic review. PLoS Med. 2006;3(9):e341 


\section{$\underline{\text { Figure Legends }}$}

Table 1. Baseline characteristics of patient hospitalized for TAVR, stratified by hospital teaching status.

TAVR: Transcatheter aortic valve replacement

PVD: Peripheral vascular disease

Figure 1. Trend in percentage of TAVR procedures performed per year between nonteaching and teaching hospitals (2011-2014).

TAVR: Transcatheter aortic valve replacement

Figure 2. Cost of hospitalization for TAVR between hospital teaching status ( $p$ for difference $=0.02$ )

USD: United States dollars

Figure 3: Rates (\%) of TAVR-associated complications

Acute MI: Acute Myocardial infarction

DVT/PE: Deep vein thrombosis/Pulmonary embolism

Figure 4. Comparing TAVR-associated complications between teaching and nonteaching hospital

DVT/PE: Deep vein thrombosis/Pulmonary embolism 